\title{
Article
}

\section{CXCL16/CXCR6 Axis in Adipocytes Differentiated from Human Adipose Derived Mesenchymal Stem Cells Regulates Macrophage Polarization}

\author{
Seung-Cheol Lee ${ }^{1}\left(\mathbb{D}\right.$, Yoo-Jung Lee ${ }^{1}$, Inho Choi ${ }^{2}$, Min Kim ${ }^{1}$ and Jung-Suk Sung ${ }^{1, *}$ (I) \\ 1 Department of Life Science, Dongguk University-Seoul, Goyang 10326, Korea; \\ hen3420@dongguk.edu (S.-C.L.); wjdyd85@naver.com (Y.-J.L.); pipikimmin@dongguk.edu (M.K.) \\ 2 Department of Pharmaceutical Engineering, Hoseo University, Asan 31499, Korea; inhochoi@hoseo.edu \\ * Correspondence: sungjs@dongguk.edu; Tel.: +82-31-961-5132; Fax: +82-31-961-5108
}

check for updates

Citation: Lee, S.-C.; Lee, Y.-J.; Choi, I.; Kim, M.; Sung, J.-S.

CXCL16/CXCR6 Axis in Adipocytes Differentiated from Human Adipose Derived Mesenchymal Stem Cells Regulates Macrophage Polarization. Cells 2021, 10, 3410. https://doi.org/ 10.3390/cells10123410

Academic Editors: Mehdi Najar and Christian Dani

Received: 2 November 2021

Accepted: 2 December 2021

Published: 3 December 2021

Publisher's Note: MDPI stays neutral with regard to jurisdictional claims in published maps and institutional affiliations.

Copyright: (C) 2021 by the authors. Licensee MDPI, Basel, Switzerland. This article is an open access article distributed under the terms and conditions of the Creative Commons Attribution (CC BY) license (https:/ / creativecommons.org/licenses/by/ $4.0 /)$.

\begin{abstract}
Adipocytes interact with adipose tissue macrophages (ATMs) that exist as a form of M2 macrophage in healthy adipose tissue and are polarized into M1 macrophages upon cellular stress. ATMs regulate adipose tissue inflammation by secreting cytokines, adipokines, and chemokines. CXC-motif receptor 6 (CXCR6) is the chemokine receptor and interactions with its specific ligand CXCmotif chemokine ligand 16 (CXCL16) modulate the migratory capacities of human adipose-derived mesenchymal stem cells (hADMSCs). CXCR6 is highly expressed on differentiated adipocytes that are non-migratory cells. To evaluate the underlying mechanisms of CXCR6 in adipocytes, THP-1 human monocytes that can be polarized into M1 or M2 macrophages were co-cultured with adipocytes. As results, expression levels of the M1 polarization-inducing factor were decreased, while those of the M2 polarization-inducing factor were significantly increased in differentiated adipocytes in a co-cultured environment with additional CXCL16 treatment. After CXCL16 treatment, the anti-inflammatory factors, including p38 MAPK ad ERK1/2, were upregulated, while the proinflammatory pathway mediated by Akt and NF- $\mathrm{KB}$ was downregulated in adipocytes in a cocultured environment. These results revealed that the CXCL16/CXCR6 axis in adipocytes regulates M1 or M2 polarization and displays an immunosuppressive effect by modulating pro-inflammatory or anti-inflammatory pathways. Our results may provide an insight into a potential target as a regulator of the immune response via the CXCL16/CXCR6 axis in adipocytes.
\end{abstract}

Keywords: hADMSCs; adipocytes; cell-cell interaction; chemokines; CXCL16/CXCR6 axis; macrophage polarization

\section{Introduction}

Adipose tissue, which is generally recognized as a complex metabolic organ, produces various factors which have important endocrine functions, including cytokines, chemokines, and adipokines, such as leptin and adiponectin [1,2]. Of these factors, circulating inflammatory chemokines are strongly associated with obesity, which is a state of chronic low-grade systemic inflammation, and the development of allergic or immune diseases as a result of their interaction with macrophages [3,4]. In particular, adipose tissue macrophages (ATMs) play a crucial role in crosstalk with adipocytes by modulating their localization and inflammatory features [5-7]. ATMs can be classified into M1 or M2 macrophages, which are formed via the influence of the various innate and adaptive immune cells involved in the regulation of adipose tissue inflammation under different adipocyte conditions [6,8-10]. Classically activated M1 macrophages are stimulated by Th1 cells, CD8 cells, and mast cells secreting interferon- $\delta$ (IFN- $\delta$ ), tumor necrosis factor- $\alpha$ (TNF- $\alpha$ ), interleukin-6 (IL-6), and C-C motif chemokine ligand 2 (CCL2). They contribute to the inflammatory response of neighboring cells, particularly in adipocytes displaying a crown-like dead structure [11,12]. In contrast, alternatively activated M2 macrophages 
are distributed in healthy adipose tissue via the expression of anti-inflammatory factors, such as fibronectin, cluster of differentiation 206 (CD 206), C-C motif chemokine ligand 22 (CCL22), and interleukin-10 (IL-10). They are induced by Th2 cytokines, which are involved in the repair or remodeling of adipose tissue [13-15]. Research into M1/M2 paradigm has provided a useful framework with which to alter adipocyte function as an inflammatory effector because macrophages are considered to be key regulatory cells in the control of adipose tissue inflammation via the secretion of cytokines and chemokines [16-18].

Chemokine receptors are G protein-coupled receptors (GPCR) classified into four classes and named according to the ligand they interact with [19]. The regulation of chemokine receptors and their downstream signaling pathways are considered promising molecular targets since they modulate various biological functions, such as cell migration, cancer metastasis, and angiogenesis [20]. Among various chemokine receptors, CXCmotif receptor 6 (CXCR6) is known to induce the recruitment of $\mathrm{T}$ cells that participate in chronic inflammation, the invasion of glial precursor cells, and in the migration of mesenchymal stem cells via the interaction of its specific ligand CXC-motif chemokine ligand 16 (CXCL16) [21-23].

Human mesenchymal stem cells, first discovered in non-hematopoietic stem cell populations, are multipotent stem cells that differentiate into osteoblasts, chondrocytes, neuronal cells, and adipocytes [24]. They migrate into injured tissue in response to signals sent in certain in physiological circumstances and differentiate into local components of injured tissue, secreting various types of growth factors and cytokines $[25,26]$. It has widely been known that the homing and migration of mesenchymal stem cells are mediated by various cytokines, growth factors, and chemokines upon interaction with their receptors, including CXCR6 [22,27]. While the functional role of the CXCL16/CXCR6 axis on the migratory capacity is intensively studied in mesenchymal stem cells that tend to migrate into certain tissues as part of cellular responses, the function of CXCR6 on differentiated adipocytes that tend to remain in their original location has yet to be elucidated [28].

Our previous report showed that CXCR6 is highly expressed in adipocytes differentiated from human adipose derived mesenchymal stem cells (hADMSCs) [29]. In this study, we further evaluated and clarified the role of CXCR6 in adipocytes interacting with macrophages and its underlying molecular mechanisms.

Here, differentiated adipocytes expressing CXCR6 were co-cultured with THP-1 cells, which were polarized into M1 or M2 macrophages, with or without additional CXCL16 treatment, which is the specific ligand of CXCR6. It is important to determine whether adipocytes interact with macrophages by expressing pro-inflammatory or antiinflammatory factors on the molecular level in response to additional CXCL16 treatment, since focusing on the crosstalk between the chemokines and the macrophages in adipose tissue can aid in the understanding of the mechanisms associated with the modulatory effects of adipose tissue inflammation [30-33].

\section{Materials and Methods}

\subsection{Cell Cultures and the Adipogenic Differentiation of hADMSCs}

The human mesenchymal cell line, hADMSCs, were purchased from CEFO (Seoul, Korea) and cultured in hADMSC growth medium (CEFO, Seoul, Korea) until cells were $80 \%$ confluent. To induce adipogenic differentiation, hADMSCs of biological passage number 4 were used. Cells were detached using Accutase (Innovative Cell Technologies, San Diego, CA, USA) and then seeded into appropriate cell culture dishes at the density of 6000 cells $/ \mathrm{cm}^{2}$ and cultured until they become $80 \%$ confluent. Then, differentiation into adipocytes was induced by culturing the cells for 18 days in high-glucose Dulbecco's Modified Eagle Medium (DMEM) (Gibco, Amarillo, TX, USA) containing 1\% penicillin streptomycin (Gibco, Amarillo, TX, USA), 10\% fetal bovine serum (Alphabioregen, Boston, MA, USA), $1 \mu$ M dexamethasone (Sigma-Aldrich Chemical, St. Louis, MO, USA), $100 \mu \mathrm{M}$ indomethacin (Sigma-Aldrich Chemical, St. Louis, MO, USA), $10 \mu \mathrm{g} / \mathrm{mL}$ insulin (Welgene, Gyeongsan, Korea), and $500 \mu \mathrm{M}$ 3-isobutyl-1-methylxanthine (Sigma-Aldrich Chemical, 
St. Louis, MO, USA). The differentiation medium was replaced every 3 days. Differentiated adipocytes were validated by the relative expression of mature adipocyte markers and the stained cellular lipid droplets in differentiated adipocytes were compared with hADMSCs (Figure S1).

\subsection{RNA Extraction and Real-Time PCR}

RNA was isolated using Trizol (Invitrogen, Waltham, MA, USA), following the manufacturer's protocol. cDNA was synthesized from $0.8 \mu \mathrm{g}$ of total RNA using Reverse Transcription Master Premix (ELPIS, Daejeon, Korea) according to the manufacturer's instructions. To amplify the cDNA, 40 cycles of PCR were performed using SYBR Green PCR Master Mix (KAPA Biosystem, Wilmington, MA, USA), according to the manufacturer's protocol. The expression levels of each target gene were normalized to GAPDH as an internal control. The specific oligonucleotide primers used in the study are listed in Table 1.

Table 1. List of oligonucleotide primers used in the study.

\begin{tabular}{|c|c|c|}
\hline Gene Name & & Sequences \\
\hline \multirow{2}{*}{$T N F-\alpha$} & Forward & AGAGAAGCCAACTACAGACC \\
\hline & Reverse & CAGTATGTGAGAGGAAGAGAA \\
\hline \multirow{2}{*}{$I L-6$} & Forward & CAGAACAGATTTGAGAGTAGTGA \\
\hline & Reverse & CGCAGAATGAGATGAGTTGT \\
\hline \multirow{2}{*}{$I L-1 \beta$} & Forward & GGCTTATTACAGTGGCAATG \\
\hline & Reverse & TAGTGGTGGTCGGAGATT \\
\hline \multirow{2}{*}{$I L-8$} & Forward & GAAGGAACCATCTCACTGT \\
\hline & Reverse & ССАСТСТСААТСАСТСТСА \\
\hline \multirow{2}{*}{ Fibronectin } & Forward & TCATCCGTGGTTGTATCA \\
\hline & Reverse & GTGGTCTCAGTAGCATCT \\
\hline \multirow{2}{*}{ CCL22 } & Forward & AAGGCAGTTACATATCAATACAG \\
\hline & Reverse & GAGGCAGAGGCTTCAATA \\
\hline \multirow{2}{*}{ CD206 } & Forward & CGGAGTAGTCATCATTGTG \\
\hline & Reverse & CGAGTGTTCATTCTGTTCA \\
\hline \multirow{2}{*}{$I L-10$} & Forward & AAGCCTTGTCTGAGATGAT \\
\hline & Reverse & CCTTGATGTCTGGGTCTT \\
\hline \multirow{2}{*}{ CCL2 } & Forward & CGAGAGGCTGAGACTAAC \\
\hline & Reverse & GAAGGTGGCTGCTATGAG \\
\hline \multirow{2}{*}{$M-C S F$} & Forward & GAAGGAGGACCAGCAAGT \\
\hline & Reverse & CAGCAAGACCAGGATGAC \\
\hline \multirow{2}{*}{$I L-13$} & Forward & ATCCGATCCTCAATCCTC \\
\hline & Reverse & CTGGTTCTGGGTGATGTT \\
\hline \multirow{2}{*}{ FABP4 } & Forward & TCAAGAGCACCATAACCTT \\
\hline & Reverse & TTCCACCACCAGTTTATCA \\
\hline \multirow{2}{*}{ Adiponectin } & Forward & ACCACTATGATGGCTCCACT \\
\hline & Reverse & GGTGAAGAGCATAGCCTTGT \\
\hline \multirow{2}{*}{$P P A R \gamma$} & Forward & CGAAGACATTCCATTCACAA \\
\hline & Reverse & CACAGACACGACATTCAAT \\
\hline \multirow{2}{*}{ GAPDH } & Forward & TATGACAACAGCCTCAAGAT \\
\hline & Reverse & GAGTCCTTCCACGATACC \\
\hline
\end{tabular}

\subsection{Western Blot}

The differentiated adipocytes were collected using an RIPA buffer (Biosolution, Seoul, Korea) containing a protease or phosphatase inhibitor (Sigma-Aldrich Chemical, St. Louis, MO, USA) and then centrifuged at 16,200 rpm for $30 \mathrm{~min}$. Cell extracts were quantified using a BCA protein assay kit (Pierce Biotechnology, Waltham, MA, USA), according 
to the manufacturer's instructions. Approximately 30-40 $\mu \mathrm{g}$ of protein was separated using $10 \%$ sodium dodecyl sulfate polyacrylamide gel electrophoresis (SDS-PAGE) and transferred to polyvinlylidene difluoride membranes (GE Healthcare, Chicago, IL, USA) for western blot analysis. At first, the transferred membrane was blocked with $1 X$ Trisbuffered saline with Tween 20 (Sigma-Aldrich Chemical, St. Louis, MO, USA) containing $5 \%$ skimmed milk (BD Biosciences, Franklin Lakes, NJ, USA) for $1 \mathrm{~h}$ and then incubated with the primary antibodies p-Akt, t-Akt, p-p38, t-p38, p-JNK, t-JNK, and t-ERK1/2 (Cell Signaling Technology, Danvers, MA, USA), and p-NF-kB, t-NF- $\mathrm{B}$, and p-ERK1/2 (Santa Cruz Biotechnology, Santa Cruz, CA, USA) at appropriate dilutions in 1X TBST containing $1 \%$ skimmed milk overnight at $4{ }^{\circ} \mathrm{C}$. The membrane was washed three times with $1 \mathrm{X}$ TBST for $10 \mathrm{~min}$ and incubated with secondary anti-rabbit (Cell Signaling Technology, Danvers, MA, USA) and anti-mouse (Santa Cruz Biotechnology, Santa Cruz, CA, USA) antibodies in $1 \times$ TBST containing $1 \%$ skimmed milk for $45 \mathrm{~min}$. The membrane was also washed three times for $15 \mathrm{~min}$ and the images were visualized using an enhanced chemiluminescence detection reagent (GE Healthcare, Chicago, IL, USA) and a Chemi-Doc (Bio-Rad Laboratories, Hercules, CA, USA) imaging device.

\subsection{Enzyme-Linked Immunosorbent Assay (ELISA)}

Secretion levels of soluble TNF- $\alpha$ and IL-10 in co-cultured THP-1 cells and differentiated adipocytes with CXCL16 treatment were determined using human TNF- $\alpha$ and an IL-10 ELISA kit (BioLegend, San Diego, CA, USA), according to the manufacturer's instructions.

\subsection{THP-1 Cell Differentiation}

THP-1 human monocytes were purchased from ATCC (Manassas, VA, USA) and cultured in RPMI1640 (Welgene, Gyeongsan, Korea) containing 10\% FBS, 2 mM L-glutamine, and $1 \%$ penicillin streptomycin. THP-1 cells were differentiated into M0 macrophages using phorbol 12-myristate 13-acetate (Sigma-Aldrich Chemical, St. Louis, MO, USA) for $24 \mathrm{~h}$ and then polarized to M1 or M2 macrophages using $20 \mathrm{ng} / \mathrm{mL}$ of LPS (Sigma-Aldrich Chemical, St. Louis, MO, USA) or $20 \mathrm{ng} / \mathrm{mL}$ of IL-4 and IL-13 (PeproTech, Rocky Hill, NJ, USA), respectively, for $48 \mathrm{~h}$.

\subsection{Polarization of THP-1 Cells Co-Cultured with Differentiated Adipocytes}

THP-1 human monocytes were seeded in a $0.4 \mu \mathrm{m}$ transwell insert (Corning Inc, Corning, NY, USA) at a density of $3 \times 10^{5}$ cells/well with PMA and then added to a 6-well plate with differentiated adipocytes with or without CXCL16 treatment for $24 \mathrm{~h}$. M0 macrophages co-cultured with adipocytes were polarized into M1 or M2 macrophages with LPS or IL-4 and IL-13 treatment, respectively, w/wo CXCL16 treatment for $48 \mathrm{~h}$. The M0, M1, and M2 macrophages were extracted to evaluate the levels of gene expression exhibited by M1 or M2 polarization markers with additional CXCL16 treatment.

\subsection{Oil Red O Staining of Differentiated Adipocytes}

Cells were washed twice with PBS and fixed with 10\% formalin (Sigma-Aldrich Chemical, St. Louis, MO, USA) for $10 \mathrm{~min}$ on ice. After fixation, cells were washed with PBS and 60\% isopropanol (Sigma-Aldrich Chemical, St. Louis, MO, USA) and air dried. Cells were stained with Oil Red O (Sigma-Aldrich Chemical, St. Louis, MO, USA) at room temperature for $30 \mathrm{~min}$ and were washed twice with distilled water. Stained lipid droplets were imaged using microscopy (Nikon, Tokyo, Japan). To quantify the relative lipid contents in cells, $100 \%$ isopropanol was used to dissolve the dye and the absorbance was measured at $492 \mathrm{~nm}$ with a microplate reader (Tecan, Männedorf, Switzerland).

\subsection{Statistical Analysis}

The statistical analysis was performed using GraphPad Prism 5.0 (GraphPad Software Inc., San Diego, CA, USA). The results were expressed as the mean \pm standard error of 
at least three independent experiments. Multiple comparisons were conducted using a one-way ANOVA with the Bonferroni method. Comparisons between two samples were made using by $t$-tests.

\section{Results}

\subsection{Validation of THP-1 Cell Polarization into M1 or M2 Macrophages}

Under different conditions, ATMs can polarize into M1 or M2 macrophages. In lean adipose tissue, M2 ATMs promote adipocyte survival leading to adipose tissue hyperplasia and the regulation of insulin sensitivity by interacting with adipocytes to maintain energy homeostasis. In contrast, M1 ATMs, which are induced by oxidative stress, trigger an inflammatory response in neighboring cells in obese adipose tissue $[8,9,13]$.

To evaluate the interaction between adipocytes and ATMs, we used THP-1 human monocytes that express specific markers in a similar way to freshly isolated cells and that can polarize into M1 or M2 macrophages by LPS and IL-4 and IL-13 treatment, respectively [34,35]. The polarization of THP-1 cells into M1 or M2 macrophages was validated by assessing the gene expression of M1 and M2 polarization-specific markers using real-time RT-PCR (Figure 1). Human monocyte THP-1 cells were differentiated into M0 macrophages with PMA treatment for $24 \mathrm{~h}$ and these were then polarized to M1 or M2 macrophages with a treatment of $20 \mathrm{ng} / \mathrm{mL}$ LPS or $20 \mathrm{ng} / \mathrm{mL}$ IL-4 and IL-13, respectively, for $48 \mathrm{~h}$. The levels of gene expression for the M1 and M2 macrophage markers were evaluated for the validation of THP- 1 cell polarization into M1 or M2 macrophages. The expression levels of M1 macrophage markers (TNF- $\alpha$, IL-1 $\beta$, IL-6, and IL-8) were significantly higher in M1 macrophages with LPS treatment as compared with M2 macrophages (Figure 1A). In addition, the levels of gene expression for the M2 macrophage markers (fibronectin, CCL22, CD206, and IL-10) were significantly upregulated in M2 macrophages with IL-4 and IL-13 treatment as compared with M1 macrophages (Figure 1B). Based on these results, THP-1 differentiation into M0, M1, and M2 macrophages was validated for further experiments.

\subsection{Expression Levels of M1 and M2 Polarization Markers in THP-1 Cells Co-Cultured with Differentiated Adipocytes upon Additional CXCL16 Treatment}

To examine whether adipocytes interact with THP-1 cells and affect M1 or M2 polarization by CXCL16 treatment, THP-1 cells were co-cultured with adipocytes differentiated from hADMSCs and then differentiated into M0, M1, and M2 macrophages.

We first measured the expression levels of M1 and M2 polarization markers in M0, M1, and M2 macrophages upon CXCL16 treatment. As shown in Figure 2A, the expression levels of M1 polarization markers (TNF- $\alpha$, IL-8) were slightly decreased. Notably, the expression levels of M2 polarization markers (CCL22) were slightly increased in M2 macrophages from THP-1 cells with additional CXCL16 treatment (Figure 2B). The expression levels of fibronectin and CD206 were also slightly increased, but this was not statistically significant (Figure 2B). These results indicate that additional CXCL16 treatment on THP-1 cells attenuates polarization into M1 macrophages and enhances polarization into M2 macrophages on its own. 
A
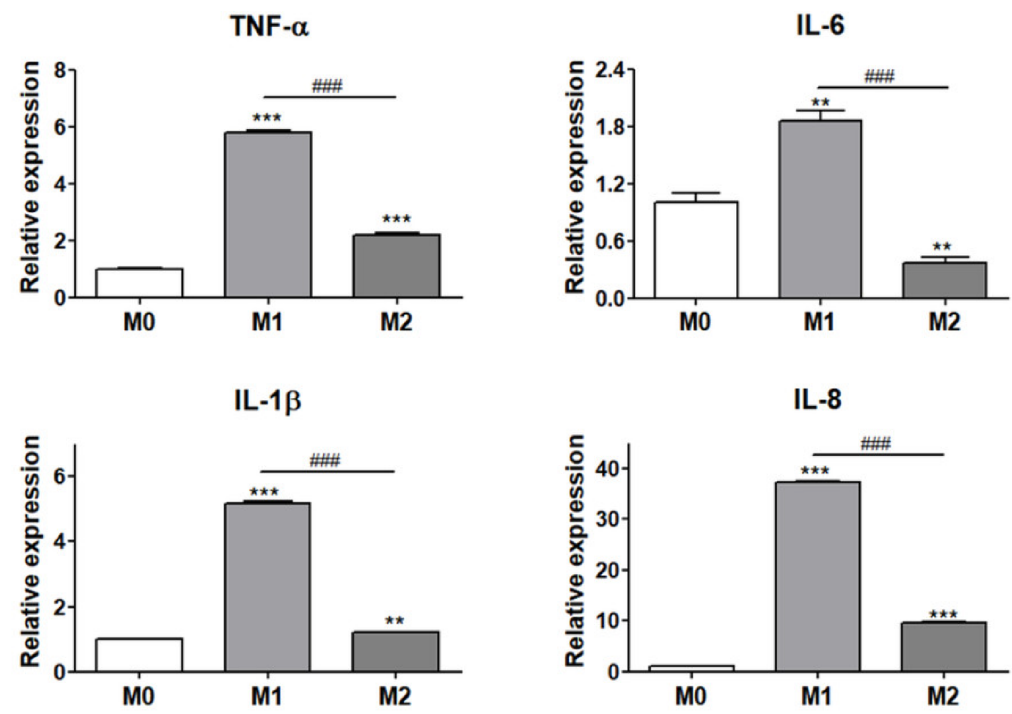

B
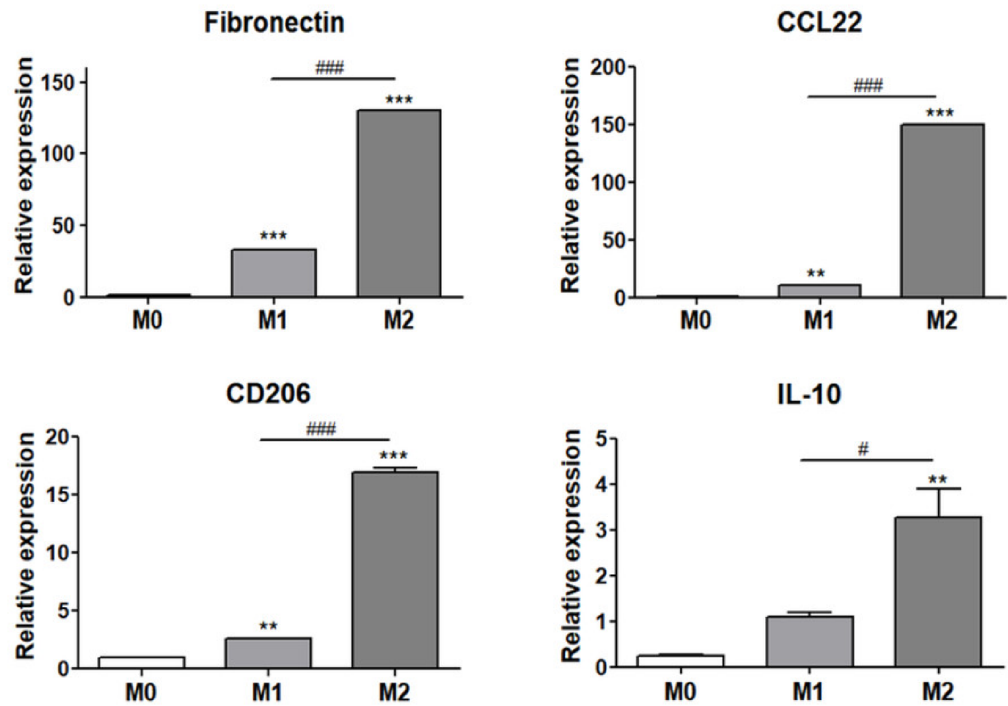

Figure 1. Validation of THP-1 cell differentiation to M1 or M2 macrophages. (A) Gene expression levels of M1 polarization markers (TNF- $\alpha$, IL-6, IL-1 $\beta$, and IL-8) and (B) M2 polarization markers (fibronectin, CD206, IL-10, and CCL22). ${ }^{* *} p<0.01$ and ${ }^{* *} p<0.001$ indicate statistically significant differences compared with the M0 group. $n=4$ trials per samples and control. ${ }^{\#} p<0.05$ and \#\#\# $p<0.001$ indicate statistically significant differences between M1 and M2 macrophages.

We then examined the expression levels of M1 and M2 polarization markers in THP-1 cells co-cultured with differentiated adipocytes with additional CXCL16 treatment. The expression levels of the M1 macrophages markers (TNF- $\alpha$, IL-1 $\beta$, and IL-8) were significantly decreased with additional CXCL16 treatment (Figure 2C). In contrast, the expression levels of the M2 macrophage markers (fibronectin, CD206, and CCL22) were significantly increased with additional CXCL16 treatment (Figure 2D). 

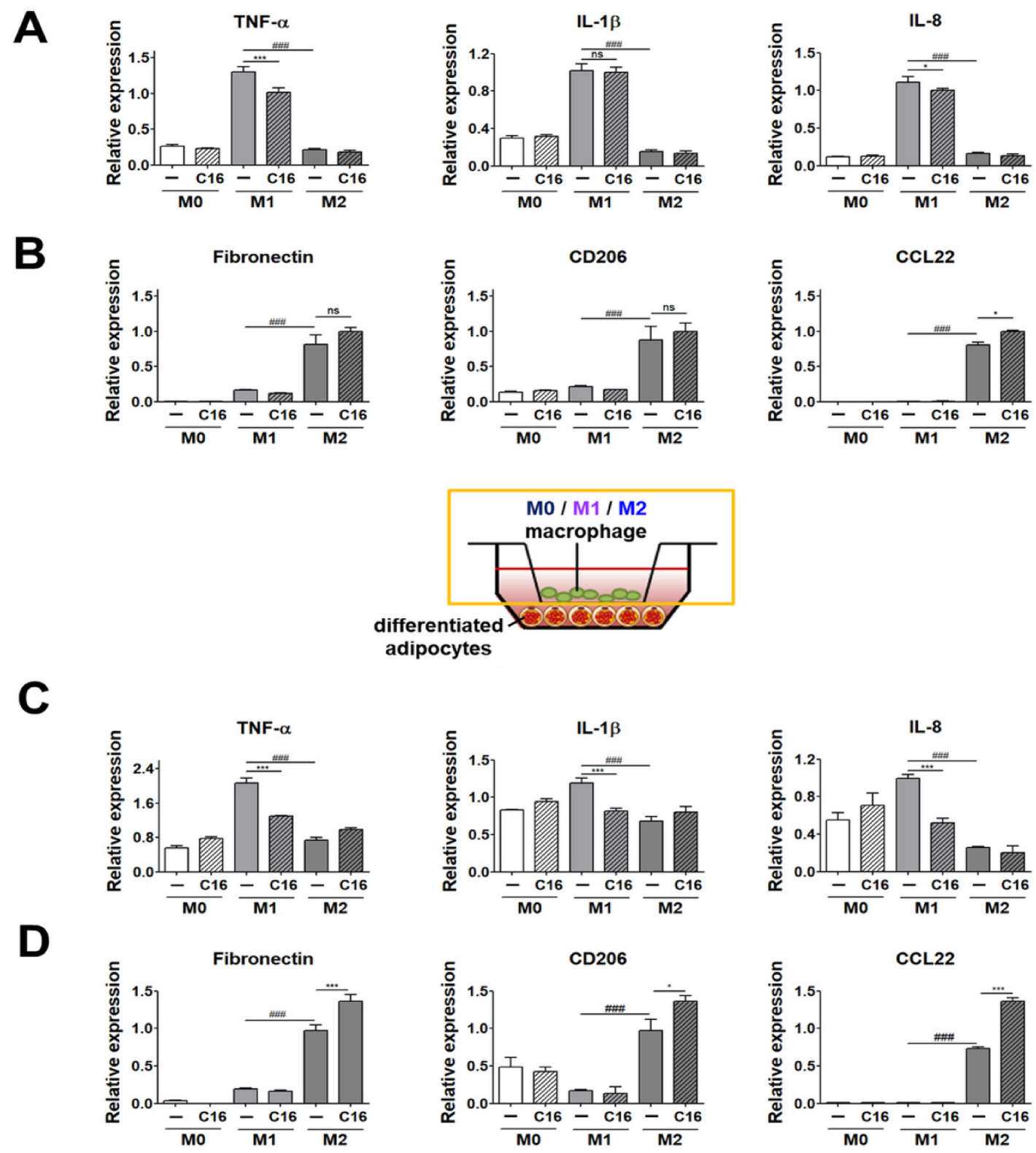

Figure 2. Expression levels of M1 and M2 polarization markers in THP-1 cells co-cultured with differentiated adipocytes upon additional CXCL16 treatment. (A) Gene expression levels of M1 polarization markers (TNF- $\alpha$, IL-1 $\beta$, and IL-8) and (B) M2 polarization markers (fibronectin, CD206, and CCL22) in THP-1 cells upon CXCL16 treatment. $n=3$ trials per samples and control. (C) Gene expression levels of M1 polarization markers (TNF- $\alpha$, IL-1 $\beta$, and IL-8) and (D) M2 polarization markers (fibronectin, CD206, and CCL22) in THP-1 cells co-cultured with differentiated adipocytes upon additional CXCL16 treatment. $n=5$ trials per samples and control. ${ }^{*} p<0.05$ and ${ }^{* * *} p<0.001$ compared with macrophages without CXCL16 treatment. ${ }^{\# \#} p<0.001$ indicate statistically significant differences between M1 and M2 macrophages. Not significant, ns.

\subsection{Expression Levels of M1 and M2 Polarization-Inducing Factors on Differentiated Adipocytes upon Additional CXCL16 Treatment}

The gene expression levels of M1-inducing factors (CCL2, IL-6, and TNF- $\alpha$ ) and M2inducing factors (M-CSF, IL-10, and IL-13) were evaluated in differentiated adipocytes co-cultured with THP-1 cells to examine whether the expression of M1- and M2-inducing factors in co-cultured adipocytes affected M1 or M2 polarization upon additional CXCL16 treatment. 
We analyzed the levels of M1 and M2 polarization-inducing factors in differentiated adipocytes in the environment without THP- 1 cells. As shown in Figure 3A, there was no significant difference in the expression levels (CCL2, M-CSF, IL-10, and IL-13) of M1 or M2 polarization-inducing factors in differentiated adipocytes in the absence of THP-1 cells upon CXCL16 treatment. This indicates that CXCL16 treatment of adipocytes cultured without macrophages does not display a potential to affect the M1 or M2 polarization of THP-1 cells.

CCL2

A
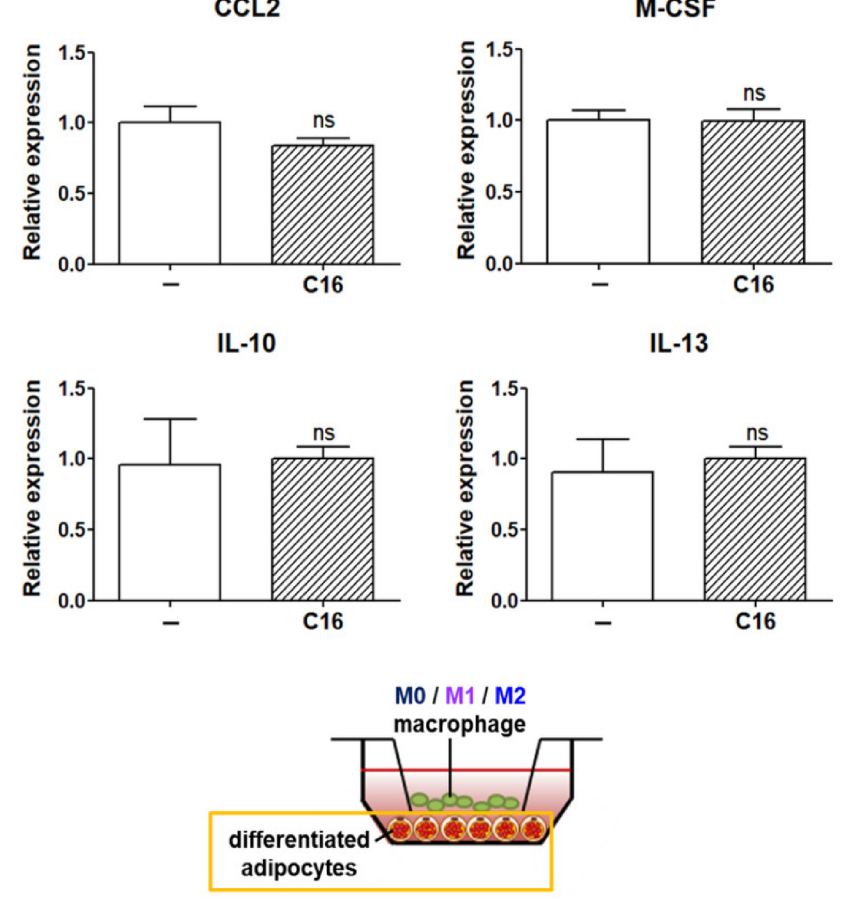

B
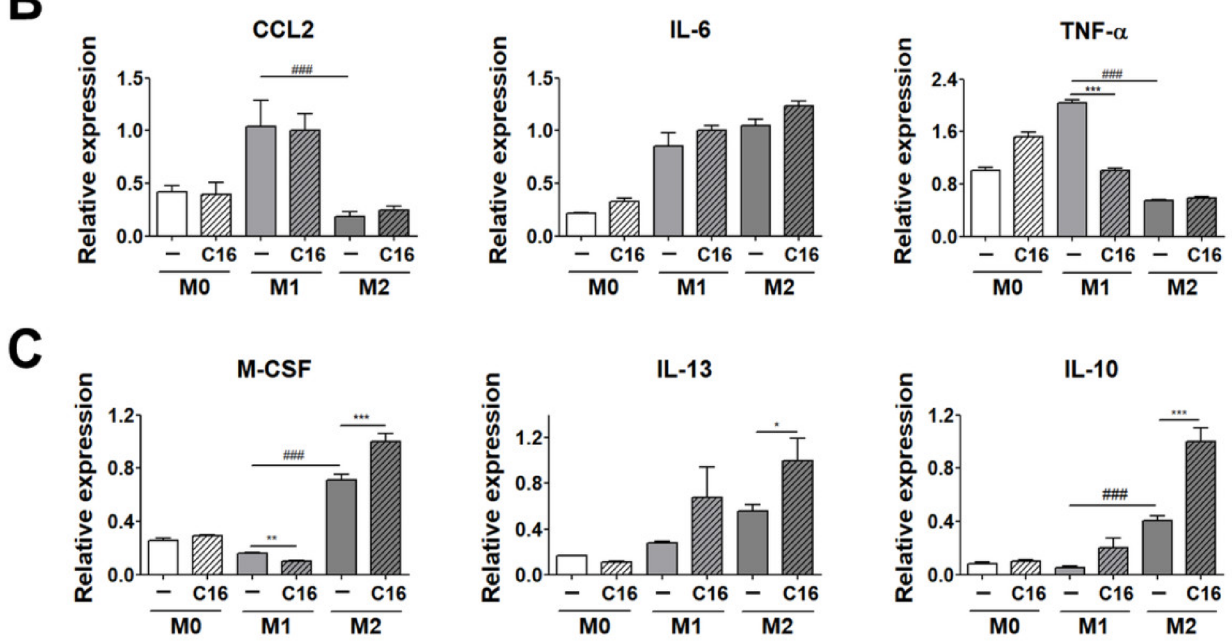

Figure 3. Expression levels of M1 and M2 polarization-inducing factors on differentiated adipocytes upon additional CXCL16 treatment. (A) Gene expression levels of M1 polarization-inducing factors (CCL2) and M2 polarization-inducing factors (M-CSF, IL-10, and IL-13) in adipocytes upon CXCL16 treatment. $n=3$ trials per samples and control. (B) Gene expression levels of M1 polarization-inducing factors (CCL2, IL-6, and TNF- $\alpha$ ) and (C) M2 polarization-inducing factors (M-CSF, IL-10, and IL-13) in differentiated adipocytes co-cultured with THP-1 cells upon additional CXCL16 treatment. $n=5$ trials per samples and control. ${ }^{*} p<0.05,{ }^{* *} p<0.01$ and ${ }^{* * *} p<0.001$ compared with adipocytes without CXCL16 treatment. $\#$ \#\# $p<0.001$ indicate statistically significant differences between adipocytes co-cultured with M1 or M2 macrophages. Not significant, ns. 
However, interestingly, the expression levels of M1 or M2 polarization-inducing factors in differentiated adipocytes were highly regulated in the co-culture environment with THP- 1 cells that had been polarized into M1 or M2 macrophages (Figure 3B,C). The expression levels of M1 polarization-inducing factors (IL- 6 and TNF- $\alpha$ ) were significantly decreased (Figure 3B), while those of M2 polarization-inducing factors (M-CSF, IL-10, and IL-13) in differentiated adipocytes significantly increased with additional CXCL16 treatment (Figure 3C).

3.4. The Secretion Levels of TNF- $\alpha$ and IL-10 in Cell Culture Media of THP-1 Cells and THP-1 Cells Co-Cultured with Differentiated Adipocytes

We examined the secretion levels of the M1 and M2 polarization-inducing factors (TNF- $\alpha$, IL-10), both of which exhibited significant differences in expression levels in adipocytes co-cultured with THP-1 cells (Figure 4).

A

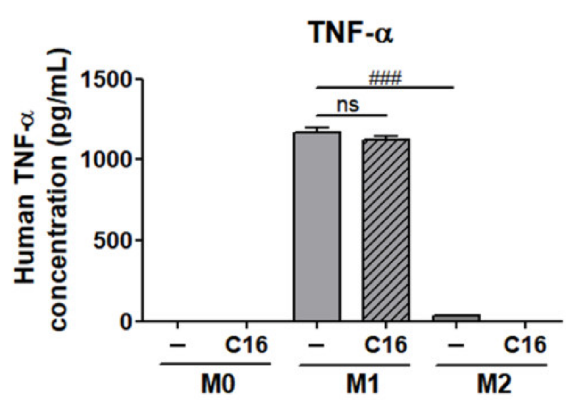

C

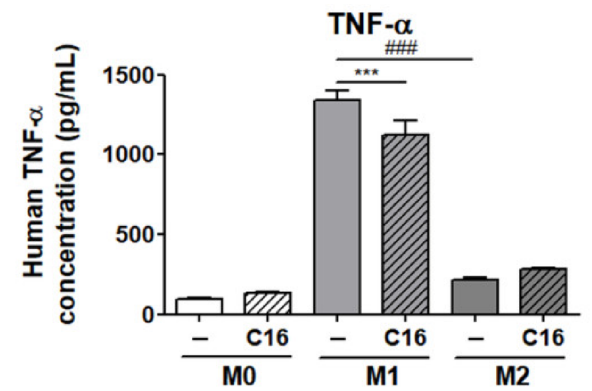

B

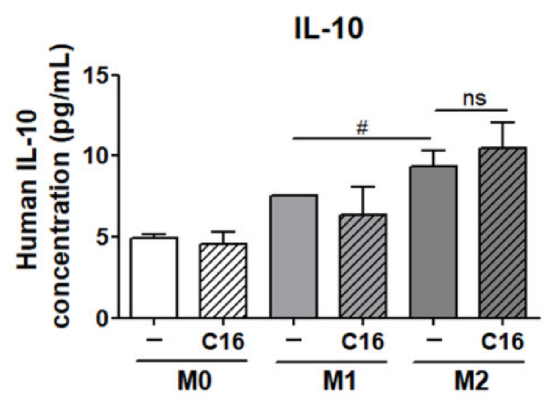

D

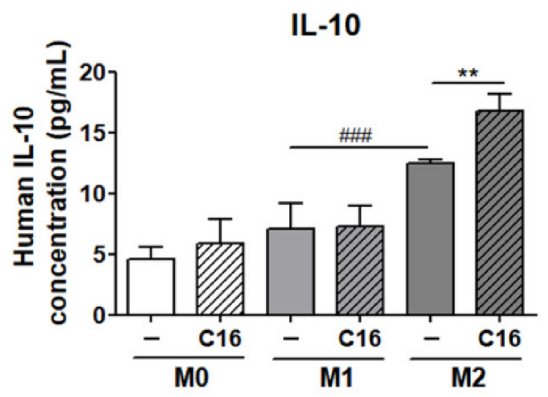

Figure 4. The secretion levels of TNF- $\alpha$ and IL-10 in cell culture media of THP-1 cells and THP-1 cells co-cultured with differentiated adipocytes. (A) Secretion levels of TNF- $\alpha$ and (B) IL-10 in a cell culture medium in THP-1 cells and the secretion levels of (C) TNF- $\alpha$ and (D) IL-10 in a cell culture medium of co-cultured THP-1 cells upon additional CXCL16 treatment. $n=5$ trials per samples and control. ${ }^{* *} p<0.01$ and ${ }^{* * *} p<0.001$ compared with samples without CXCL16 treatment. ${ }^{\#} p<0.05$ and ${ }^{\# \# \#} p<0.001$ indicate statistically significant differences between M1 and M2 macrophages. Not significant, ns.

As shown in Figure 4A,B, the secretion level of TNF- $\alpha$ and IL-10 in cell culture media were not differed with additional CXCL16 treatment in M1 and M2 macrophages from THP-1 cells. Notably, the secretion levels of TNF- $\alpha$ and IL-10 in a co-cultured media of differentiated adipocytes and THP- 1 cells were significantly decreased and increased, respectively, consistent with the data shown in Figure 3B,C (Figure 4C,D). These results suggest that CXCL16 interacts with CXCR6, which is highly expressed in differentiated adipocytes, and then regulates the polarization of THP- 1 cells into M1 or M2 macrophages via the secretion of factors including TNF- $\alpha$ and IL-10. 


\subsection{Inflammatory Signaling Pathways in Differentiated Adipocytes Co-Cultured with THP-1 Cells upon Additional CXCL16 Treatment}

To evaluate which inflammatory signaling pathways are regulated in differentiated adipocytes in a co-culture environment with THP-1 cells, the expression of signaling proteins mediating the mitogen-activated protein kinase (MAPK) and nuclear factor kappalight-chain-enhancer of activated B cells (NF- $\mathrm{kB}$ ) signaling pathways were examined (Figure 5). The nuclear factor NF- $\kappa B$ pathway has long been considered a pro-inflammatory signaling pathway and the MAPK pathway serves as an anti-inflammatory signal that suppresses the expression of NF- $\mathrm{kB}$-dependent inflammatory genes for anti-inflammatory purposes [36-38].

A

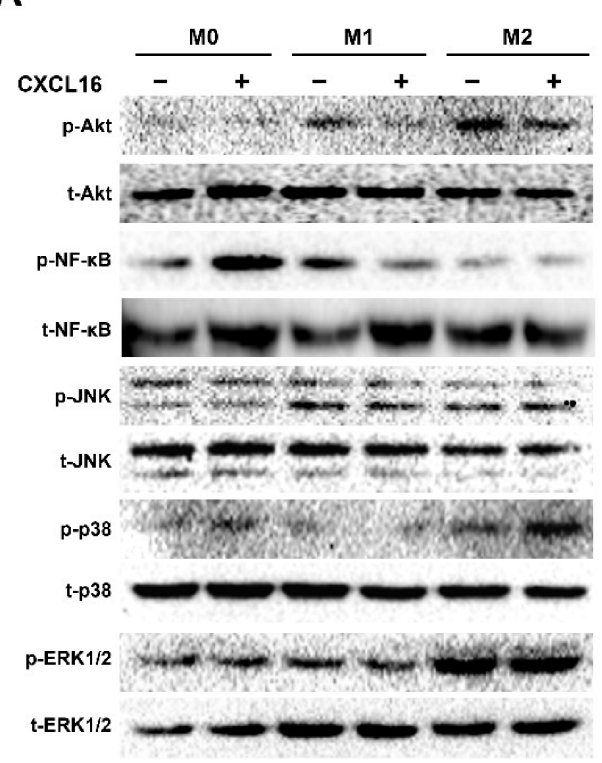

B
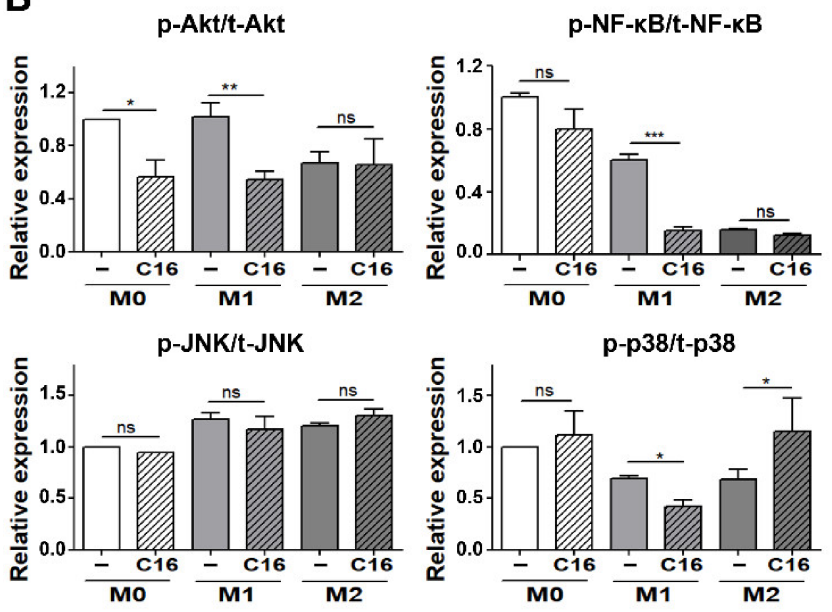

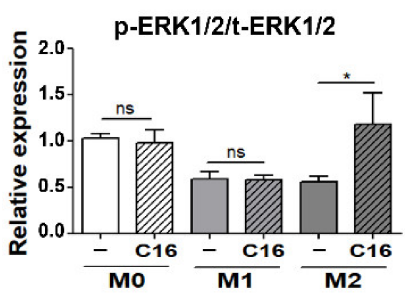

Figure 5. Inflammatory signaling pathways in differentiated adipocytes co-cultured with THP-1 cells upon additional CXCL16 treatment. (A) Pro-inflammatory (Akt, NF-KB) and anti-inflammatory pathways (JNK, p38, and ERK1/2) in adipocytes co-cultured with THP-1 cells following additional CXCL16 treatment for $30 \mathrm{~min}$. (B) Relative protein intensity of p-Akt/t-Akt, p-NF-kB/t-NF-kB, p-JNK/t-JNK, p-p38/t-p38, and p-ERK1/2/t-ERK1/2 in adipocytes. $n=3$ trials per samples and control. ${ }^{*} p<0.05,{ }^{* *} p<0.01$, and ${ }^{* * *} p<0.001$ indicate statistically significant differences. Not significant, ns.

The activation of the Akt/NF- $\mathrm{kB}$ pathway significantly decreased in differentiated adipocytes in the presence of THP-1 cells polarized into M1 macrophages with additional CXCL16 treatment. In contrast, ERK1/2 and p38 MAPK, but not JNK, were activated in differentiated adipocytes in the presence of THP-1 cells polarized into M2 macrophages.

\section{Discussion}

Previously, we reported that CXCR6 was highly expressed on cell surfaces during the differentiation of hADMSCs into adipocytes [29]. Despite the discovery on the regulatory function of CXCR6 expression and the function of the CXCL16/CXCR6 axis in the migratory capacity of hADMSCs, the function of the CXCL16/CXCR6 axis in adipocytes with poor migratory capacities has been insufficiently studied $[22,28]$. Adipocytes secrete various growth factors, cytokines, and chemokines, which are related to the metabolic syndrome, insulin resistance, obesity-related disease, and immune responses [5,39-41]. They can interact with immune cells, especially ATMs that are differentially located, depending on the state of adipose tissue $[8,11,12]$. Under different conditions, ATMs can polarize into M1 
or M2 macrophages. In lean adipose tissue, M2 ATMs promote adipocyte survival leading to adipose tissue hyperplasia and the regulation of insulin sensitivity by interacting with adipocytes to maintain energy homeostasis. In contrast, M1 ATMs, which are induced by oxidative stress, trigger an inflammatory response in neighboring cells in obese adipose tissue $[8,9,13]$.

To evaluate the interaction between adipocytes and ATMs, the polarization of THP-1 cells into M1 or M2 macrophages was first validated by assessing the gene expression of M1 and M2 polarization markers (Figure 1). Gene expression levels of M1 polarization markers (IL-1 $\beta$ ) and M2 polarization markers (fibronectin and CD206) showed no significant difference in THP-1 cells with additional CXCL16 treatment (Figure 2A,B). Additionally, the gene expression levels of M1 markers (TNF- $\alpha$ and IL-8) and M2 markers (CCL22) were only slightly downregulated or upregulated, respectively, with CXCL16 treatment. Recent research has reported that a specific motif of CXCR6 expressed in THP-1 cells binds with CXCL16 and affects the recruitment or adhesion of leukocytes [42]. This suggests that CXCR6 in THP-1 cells may have the potential to regulate the expression of inflammatory factors through its interaction with CXCL16. Therefore, the slight modulation of M1 and M2 polarization of THP-1 cells following CXCL16 treatment can be interpreted as being due to the CXCL16/CXCR6 axis.

On the other hand, interestingly, the expression levels of M1 and M2 polarization markers in M1 and M2 macrophages were significantly downregulated or upregulated, respectively, upon CXCL16 treatment when they were co-cultured with differentiated adipocytes (Figure 2C,D).

These results suggest that adipocytes with CXCL16 treatment in co-cultured environments with THP-1 cells can affect the polarization potential of THP-1 cells into M1 and M2 macrophages.

Gene expression levels of M1-inducing factors (CCL2, IL-6, and TNF- $\alpha$ ) and M2inducing factors (M-CSF, IL-10, and IL-13) in differentiated adipocytes co-cultured with THP-1 cells were measured to examine whether the expression of M1- and M2-inducing factors in co-cultured adipocytes affected M1 or M2 polarization with additional CXCL16 treatment. As shown in Figure 3A, there was no statistically significant difference in the levels of CCL2, M-CSF, IL-10, and IL-13, which are basically expressed in adipocytes in physiological conditions upon CXCL16 treatment. However, interestingly, the expression levels of these factors differed significantly upon additional CXCL16 treatment when differentiated adipocytes were co-cultured with THP-1 cells that are polarized into M1 and M2 macrophages. The M1-inducing factors IL- 6 and TNF- $\alpha$ decreased upon CXCL16 treatment and the M2-inducing factors M-CSF, IL-10, and IL-13 significantly increased upon CXCL16 treatment in adipocytes co-cultured with THP-1 cells (Figure 3B,C). This suggests that CXCL16 treatment does not affect the expression of M1 or M2 inducing factors in differentiated adipocytes on its own but attenuates polarization into M1 macrophages and promotes polarization into M2 macrophages when in the co-cultured environment of adipocytes and THP-1 cells. That is, adipocytes at the location available to interact with macrophages are significantly affected by CXCL16, resulting in the modulatory expression of M1 or M2 inducing factors.

Measurement of the secretion levels of TNF- $\alpha$ and IL-10 demonstrated that they were significantly modulated in differentiated adipocytes co-cultured with THP-1 cells by additional CXCL16 treatment. Consistent with the results shown in Figure 3, secretion levels of TNF- $\alpha$ and IL-10 were significantly downregulated and upregulated, respectively, in co-cultured media of differentiated adipocytes and THP-1 cells (Figure 4C,D). These results suggest that CXCL16 interacts with CXCR6, which is highly expressed in differentiated adipocytes, and then regulates the polarization of THP-1 cells into M1 or M2 macrophages.

The MAPK pathway-p38 and ERK1/2 in particular-serves as an anti-inflammatory signal that suppresses the expression of NF-kB-dependent inflammatory genes and makes them potential targets for anti-inflammatory activity [36,37]. The NF- $\kappa \mathrm{B}$ pathway is a proinflammatory signaling pathway that involves the expression of pro-inflammatory genes, 
including cytokines, chemokines, and adhesion molecules [38]. It was found in this study that the anti-inflammatory pathway (p38MAPK, ERK1/2) was significantly activated by CXCL16 treatment in differentiated adipocytes co-cultured with THP-1 cells that had been polarized into M2 macrophages. In contrast, activation of the pro-inflammatory pathway (Akt, NF-KB) decreased with additional CXCL16 treatment when co-cultured with THP-1 cells that were polarized into M1 macrophages (Figure 5A,B).

M2 macrophages are known to produce higher levels of immunosuppressive cytokines compared to M1 macrophages, and studies showed that mesenchymal stem cells promoting M2 macrophage polarization display immunosuppressive effects [43-46]. On the basis of our observations, we can conclude that CXCL16 can modulate the characteristics of two cell populations via expression of M1 or M2 inducing factors, simultaneously modulating antior pro-inflammatory pathways in adipocytes during the interaction between adipocytes and macrophages. This indicates that CXCL16 acts as an anti-inflammatory chemokine via interaction with CXCR6, attenuating the polarization of THP-1 cells into M1 macrophages and enhancing their polarization into M2 macrophages.

Recent studies have revealed that tumor-associated macrophages (TAMs) have been shown to express an M2-like phenotype promoting tumor progression and the metastasis of cancers by upregulating CXCR6 expression [47,48]. In spite of the evidence in these studies supporting the regulatory function of macrophages on CXCR6 expression in cancer cells, the information on the function and the regulatory mechanism of CXCL16/CXCR6 in adipocytes is lacking. Therefore, further comprehensive analysis of the expression level of CXCR6 on cell surfaces and the regulation of signal transduction mediated by the CXCL16/CXCR6 axis in co-cultured environments possesses great potential. Furthermore, the discovery of the regulatory mechanism underlying the role of CXCR6 expression and the regulation of the CXCL16/CXCR6 axis would provide insights into adipose tissue microenvironments.

In summary, our results clarified the role of CXCR6, which is highly expressed in adipocytes differentiated from hADMSCs. It was found that CXCR6 interacts with CXCL16 and subsequently attenuates or enhances the pro-inflammatory or anti-inflammatory response of macrophages in the presence of adipocytes via the Akt, NF-kB or p38, and ERK1/2 pathways. We first demonstrated that CXCL16 plays a crucial role in regulating M1 or M2 polarization via interaction with neighboring ATMs. This finding promises to shed light on the development of a novel therapeutics that could regulate inflammatory responses via CXCL16/CXCR6 interaction in adipocytes with macrophages.

\section{Conclusions}

In this study, we investigated the role of CXCR6 in differentiated adipocytes in interaction with macrophages after CXCL16 treatment. In a co-cultured environment of THP-1 cells with adipocytes, polarization into M1 and M2 macrophages was attenuated and enhanced, respectively, with additional CXCL16 treatment. The polarization into M1 and M2 macrophages was significantly regulated by the expression of M1- and M2-inducing factors in differentiated adipocytes co-cultured with THP-1 cells with additional CXCL16 treatment, demonstrating that the polarization of THP-1 cells into M1 or M2 macrophages was affected by the interaction between CXCL16 and CXCR6 expressed in differentiated adipocytes. Moreover, the pro-inflammatory and anti-inflammatory pathways were modulated by CXCL16 treatment in adipocytes co-cultured with THP-1 cells. In summary, we have shown that the CXCL16/CXCR6 axis in adipocytes functions as the regulator of the inflammatory response via interactions with neighboring macrophages. These results may contribute to development of a novel stem cell therapy in the search for a potential drug target that can regulate the immune response of macrophages via theCXCL16/CXCR6 axis in adipocytes.

Supplementary Materials: The following are available online at https: / www.mdpi.com/article / 10.3390/cells10123410/s1, Figure S1: Validation of adipogenic differentiation of adipocytes from hADMSCs. 
Author Contributions: Conceptualization, S.-C.L., Y.-J.L. and J.-S.S.; investigation, S.-C.L. and Y.-J.L.; methodology, I.C., M.K. and J.-S.S.; project administration, J.-S.S.; writing-original draft, S.-C.L. and Y.-J.L.; writing-review and editing, S.-C.L. All authors have read and agreed to the published version of the manuscript.

Funding: This research was supported by a grant from the National Research Foundation of Korea (NRF) [NRF-2017R1D1A1B03030983].

Institutional Review Board Statement: Not applicable.

Informed Consent Statement: Not applicable.

Data Availability Statement: The data presented in this study are available on request from the corresponding author.

Conflicts of Interest: The authors declare no conflict of interest.

\section{References}

1. Dalmas, E.; Clement, K.; Guerre-Millo, M. Defining macrophage phenotype and function in adipose tissue. Trends Immunol. 2011, 32, 307-314. [CrossRef] [PubMed]

2. Kershaw, E.E.; Flier, J.S. Adipose tissue as an endocrine organ. J. Clin. Endocrinol. Metab. 2004, 89, 2548-2556. [CrossRef] [PubMed]

3. Mantovani, A.; Sica, A.; Sozzani, S.; Allavena, P.; Vecchi, A.; Locati, M. The chemokine system in diverse forms of macrophage activation and polarization. Trends Immunol. 2004, 25, 677-686. [CrossRef] [PubMed]

4. Sánchez-Martín, L.; Estecha, A.; Samaniego, R.; Sánchez-Ramón, S.; Ángel, V.M.; Sánchez-Mateos, P. The chemokine CXCL12 regulates monocyte-macrophage differentiation and RUNX3 expression. Blood 2011, 117, 88-97. [CrossRef]

5. Choe, S.S.; Huh, J.Y.; Hwang, I.J.; Kim, J.I.; Kim, J.B. Adipose Tissue Remodeling: Its Role in Energy Metabolism and Metabolic Disorders. Front. Endocrinol. 2016, 7, 30. [CrossRef]

6. Huh, J.Y.; Park, Y.J.; Ham, M.; Kim, J.B. Crosstalk between Adipocytes and Immune Cells in Adipose Tissue Inflammation and Metabolic Dysregulation in Obesity. Mol. Cells 2014, 37, 365-371. [CrossRef]

7. Xu, L.; Kitade, H.; Ni, Y.; Ota, T. Roles of Chemokines and Chemokine Receptors in Obesity-Associated Insulin Resistance and Nonalcoholic Fatty Liver Disease. Biomolecules 2015, 5, 1563-1579. [CrossRef]

8. Fujisaka, S.; Usui, I.; Bukhari, A.; Ikutani, M.; Oya, T.; Kanatani, Y.; Tsuneyama, K.; Nagai, Y.; Takatsu, K.; Urakaze, M.; et al. Regulatory mechanisms for adipose tissue M1 and M2 macrophages in diet-induced obese mice. Diabetes 2009, 58, $2574-2582$. [CrossRef]

9. Jablonski, K.A.; Amici, S.A.; Webb, L.M.; Ruiz-Rosado, J.D.D.; Popovich, P.G.; Partida-Sanchez, S.; De-Arellano, G.M. Novel Markers to Delineate Murine M1 and M2 Macrophages. PLoS ONE 2015, 10, e0145342.

10. Li, P.; Lu, M.; Nguyen, M.T.A.; Bae, E.J.; Chapman, J.; Feng, D.; Hawkins, M.; Pessin, J.E.; Sears, D.D.; Nguyen, A.-K.; et al. Functional heterogeneity of CD11c-positive adipose tissue macrophages in diet-induced obese mice. J. Biol. Chem. 2010, 285, 15333-15345. [CrossRef]

11. Boutens, L.; Stienstra, R. Adipose tissue macrophages: Going off track during obesity. Diabetologia 2016, 59, 879-894. [CrossRef]

12. Thomas, D.; Apovian, C. Macrophage functions in lean and obese adipose tissue. Metabolism 2017, 72, 120-143. [CrossRef]

13. Liu, C.; Li, Y.; Yu, J.; Feng, L.; Hou, S.; Liu, Y.; Guo, M.; Xie, Y.; Meng, J.; Zhang, H.; et al. Targeting the shift from M1 to M2 macrophages in experimental autoimmune encephalomyelitis mice treated with fasudil. PLoS ONE 2013, 8, e54841.

14. Nakajima, S.; Koh, V.; Kua, L.-F.; Davide, L.; Lim, K.S.; Petersen, S.H.; Yong, W.-P.; Shabbir, A.; Kono, K. Accumulation of CD11c+CD163+ Adipose Tissue Macrophages through Upregulation of Intracellular 11beta-HSD1 in Human Obesity. J. Immunol. 2016, 197, 3735-3745. [CrossRef]

15. Aron-Wisnewsky, J.; Tordjman, J.; Poitou, C.; Darakhshan, F.; Hugol, D.; Basdevant, A.; Aissat, A.; Guerre-Millo, M.; Cleément, K. Human Adipose Tissue Macrophages: M1 and M2 Cell Surface Markers in Subcutaneous and Omental Depots and after Weight Loss. J. Clin. Endocrinol. Metab. 2009, 94, 4619-4623. [CrossRef]

16. Martinez, F.O.; Gordon, S. The M1 and M2 paradigm of macrophage activation: Time for reassessment. F1000Prime Rep. 2014, 6, 13. [CrossRef]

17. Morris, D.L.; Singer, K.; Lumeng, C.N. Adipose tissue macrophages: Phenotypic plasticity and diversity in lean and obese states. Curr. Opin. Clin. Nutr. Metab. Care 2011, 14, 341-346. [CrossRef]

18. Ruytinx, P.; Proost, P.; Van Damme, J.; Struyf, S. Chemokine-Induced Macrophage Polarization in Inflammatory Conditions. Front Immunol. 2018, 9, 1930. [CrossRef]

19. UIS/WHO Subcommittee on Chemokine Nomenclature. Chemokine/chemokine receptor nomenclature. Cytokine 2003, 21, 1067-1068.

20. Ghanemi, A.; Targeting, G. Protein coupled receptor-related pathways as emerging molecular therapies. Saudi Pharm J. 2015, 23, 115-129. [CrossRef]

21. Hattermann, K.; Ludwig, A.; Gieselmann, V.; Held-Feindt, J.; Mentlein, R. The chemokine CXCL16 induces migration and invasion of glial precursor cells via its receptor CXCR6. Mol. Cell Neurosci. 2008, 39, 133-141. [CrossRef] 
22. Dhoke, N.R.; Kaushik, K.; Das, A. Cxcr6-Based Mesenchymal Stem Cell Gene Therapy Potentiates Skin Regeneration in Murine Diabetic Wounds. Mol. Ther. 2020, 28, 1314-1326. [CrossRef]

23. Lehrke, M.; Millington, S.C.; Lefterova, M.; Cumaranatunge, R.G.; Szarpary, P.; Wilenski, R.; Rader, D.J.; Lazar, M.A.; Reilly, M.P. CXCL16 is a marker of inflammation, atherosclerosis, and acute coronary syndromes in humans. J. Am. Coll. Cardiol. 2007, 49, 442-449. [CrossRef]

24. Minteer, D.; Marra, K.G.; Rubin, J.P. Adipose-Derived Mesenchymal Stem Cells: Biology and Potential Applications. In Mesenchymal Stem Cells_Basics and Clinical Application I; Springer: Berlin/Heidelberg, Germany, 2013; Volume 129, pp. 59-71.

25. Sohni, A.; Verfaillie, C.M. Mesenchymal stem cells migration homing and tracking. Stem Cells Int. 2013, 2013, 130763. [CrossRef]

26. Fu, X.; Liu, G.; Halim, A.; Ju, Y.; Luo, Q.; Song, G. Mesenchymal Stem Cell Migration and Tissue Repair. Cells 2019, 8, 784. [CrossRef]

27. Baek, S.J.; Kang, S.K.; Ra, J.C. In vitro migration capacity of human adipose tissue-derived mesenchymal stem cells reflects their expression of receptors for chemokines and growth factors. Exp. Mol. Med. 2011, 43, 596-603. [CrossRef]

28. Lustig, M.; Zadka, Y.; Levitsky, I.; Gefen, A.; Benayahu, D. Adipocytes Migration is Altered Through Differentiation. Microsc. Microanal. 2019, 25, 1195-1200. [CrossRef]

29. Lee, S.-C.; Lee, Y.-J.; Shin, M.K.; Sung, J.-S. Regulation of CXCR6 Expression on Adipocytes and Osteoblasts Differentiated from Human Adipose Tissue-Derived Mesenchymal Stem Cells. Stem Cells Int. 2020, 2020, 8870133. [CrossRef]

30. Smith, T.; Tse, M.J.; Read, E.; Liu, W.F. Regulation of macrophage polarization and plasticity by complex activation signals. Integr. Biol. 2016, 8, 946-955. [CrossRef]

31. Sarvari, A.K.; Doan-Xuan, Q.-M.; Bacso, Z.; Csomós, I.; Balajthy, Z.; Fesus, L. Interaction of differentiated human adipocytes with macrophages leads to trogocytosis and selective IL-6 secretion. Cell Death Dis. 2015, 6, e1613. [CrossRef] [PubMed]

32. Engin, A.B.; Engin, A.; Gonul, I.I. The effect of adipocyte-macrophage crosstalk in obesity-related breast cancer. J. Mol. Endocrinol. 2019, 62, R201-R222. [CrossRef] [PubMed]

33. Festuccia, W.T. Regulation of Adipocyte and Macrophage Functions by mTORC1 and 2 in Metabolic Diseases. Mol. Nutr. Food Res. 2020, 65, e1900768. [CrossRef] [PubMed]

34. Park, E.K.; Jung, H.S.; Yang, H.I.; Yoo, M.C.; Kim, C.; Kim, K.S. Optimized THP-1 differentiation is required for the detection of responses to weak stimuli. Inflamm. Res. 2007, 56, 45-50. [CrossRef]

35. Genin, M.; Clement, F.; Fattaccioli, A.; Raes, M.; Michiels, C. M1 and M2 macrophages derived from THP-1 cells differentially modulate the response of cancer cells to etoposide. BMC Cancer 2015, 15, 577. [CrossRef]

36. Kaminska, B. MAPK signalling pathways as molecular targets for anti-inflammatory therapy-from molecular mechanisms to therapeutic benefits. Biochim. Biophys. Acta (BBA) Proteins Proteom. 2005, 1754, 253-262. [CrossRef]

37. Maeng, Y.-S.; Min, J.-K.; Kim, J.H.; Yamagishi, A.; Mochizuki, N.; Kwon, J.-Y.; Park, Y.-W.; Kim, Y.-M.; Kwon, Y.-G. ERK is an anti-inflammatory signal that suppresses expression of NF-KB-dependent inflammatory genes by inhibiting IKK activity in endothelial cells. Cell. Signal. 2006, 18, 994-1005. [CrossRef]

38. Lawrence, T. The nuclear factor NF-kappaB pathway in inflammation. Cold Spring Harb. Perspect. Biol. 2009, 1, a001651. [CrossRef]

39. Ojima, K.; Oe, M.; Nakajima, I.; Muroya, S.; Nishimura, T. Dynamics of protein secretion during adipocyte differentiation. FEBS Open Bio. 2016, 6, 816-826. [CrossRef]

40. Rosen, E.D.; Spiegelman, B.M. Adipocytes as regulators of energy balance and glucose homeostasis. Nature 2006, 444, 847-853. [CrossRef]

41. Wang, P.; Mariman, E.; Renes, J.; Keijer, J. The secretory function of adipocytes in the physiology of white adipose tissue. J. Cell Physiol. 2008, 216, 3-13. [CrossRef]

42. Koenen, A.; Babendreyer, A.; Schumacher, J.; Pasqualon, T.; Schwarz, N.; Seifert, A.; Deupi, X.; Ludwig, A.; Dreymueller, D. The DRF motif of CXCR6 as chemokine receptor adaptation to adhesion. PLoS ONE 2017, 12, e0173486.

43. Das, A.; Sinha, M.; Datta, S.; Abas, M.; Chaffee, S.; Sen, C.K.; Roy, S. Monocyte and macrophage plasticity in tissue repair and regeneration. Am. J. Pathol. 2015, 185, 2596-2606. [CrossRef]

44. Guo, J.; Qiu, X.; Zhang, L.; Wei, R. Smurf1 regulates macrophage proliferation, apoptosis and migration via JNK and p38 MAPK signaling pathways. Mol. Immunol. 2018, 97, 20-26. [CrossRef]

45. Kim, J.; Hematti, P. Mesenchymal stem cell-educated macrophages: A novel type of alternatively activated macrophages. Exp. Hematol. 2009, 37, 1445-1453. [CrossRef]

46. Heo, J.S.; Choi, Y.; Kim, H.O. Adipose-Derived Mesenchymal Stem Cells Promote M2 Macrophage Phenotype through Exosomes. Stem Cells Int. 2019, 2019, 7921760. [CrossRef]

47. Lee, C.; Jeong, H.; Bae, Y.; Shin, K.; Kang, S.; Kim, H.; Oh, J.; Bae, H. Targeting of M2-like tumor-associated macrophages with a melittin-based pro-apoptotic peptide. J. Immunother. Cancer 2019, 7, 147. [CrossRef]

48. Hong, L.; Wang, S.; Li, W.; Wu, D.; Chen, W. Tumor-associated macrophages promote the metastasis of ovarian carcinoma cells by enhancing CXCL16/CXCR6 expression. Pathol. Res. Pr. 2018, 214, 1345-1351. [CrossRef] 